\title{
Geodesically linked active contours: evolution strategy based on minimal paths
}

\author{
Julien MILLE and Laurent D. COHEN \\ CEREMADE, UMR CNRS 7534, Université Paris IX-Dauphine \\ Place du Maréchal de Lattre de Tassigny, 75016 Paris, France
}

\begin{abstract}
The proposed method is related to parametric and geodesic active contours as well as minimal paths, in the context of image segmentation ${ }^{1}$. Our geodesically linked active contour model consists in a set of vertices connected by paths of minimal cost. This makes up a closed piecewise defined curve, over which an edge or region energy functional is formulated. The greedy algorithm is used to move vertices towards a configuration minimizing the energy functional. This evolution technique ensures lower sensitivity to erroneous local minima than usual gradient descent of the energy. Our method intends to take advantage of explicit active contours, minimal paths and greedy evolution techniques.
\end{abstract}

\section{Introduction}

Among well known variational models for image segmentation, active contours have drawn lively interest since their introduction by Kass et al [1]. Their key principle is the research of a curve minimizing an energy functional, which mainly depends on the adequacy of the curve to the target object. Active contours are implemented either with a parametric curve - in which case they are often referred to as 'snakes' - or in an implicit fashion based on the level set framework [2][3]. Early active contour models are mainly parametric and boundarybased, as the data term of the energy functional is an edge indicator function integrated along the curve. The Euler-Lagrange equation, determined by calculus of variations, indicates the minimizing flow to be followed by gradient descent scheme. These models are dependent of curve parameterization and unable to adapt their topology. Moreover, gradient descent is sensitive to local minima of the energy functional. Parameterization invariance is achieved by the geodesic active contour model [4], which introduces a geometrically intrinsic functional, whereas topology adaptiveness is provided by the level set implementation.

Significant attempts have been made to decrease the sensitivity to local minima, based either on the gradient descent direction or on the minimization method itself. The balloon force [5] falls into the first category, as it adds a normal-oriented inflation or retraction component, in order to increase the capture range of the snake. As regards the evolution process, several heuristics based on local searches have been proposed as alternatives to gradient descent,

\footnotetext{
1 This work was partially supported by ANR grant NanoGPSCellulaire ANR-05NANO-045-06
} 
including dynamic programming [6][7] and the greedy algorithm [8][9]. The latter, which is subsequently addressed in the paper, considers the energy as a sum of curve points energies. It basically consists in iteratively moving curve points to locations minimizing their own energies, these locations belonging to a search window. On the other hand, the minimal path approach by Cohen and Kimmel [10], which seeks for a curve of minimal cost between two end-points, can be used to recover open and closed boundaries. It is closely related to the geodesic active contour with respect to the functional to be optimized, but has in addition the main benefit of finding a global minimum efficiently thanks to the Fast Marching technique [11].

In this paper, we deal with an explicit implementation of active contour, i.e. a discrete curve defined by control points, or vertices. The described method is both related to minimal paths and greedy search. Our geodesically linked active contour model is made up of a set of vertices connected by paths of minimal cost with respect to a boundary-based metric. We define search windows centered at each vertex and evolve vertices according to a greedy fashion. Making a given vertex movable and the other ones still, we consider every geodesically linked contour passing through the points in the window of the moving vertex. This last one is finally moved to the location leading to the contour of smallest energy. The motivation for this work resides in several points. Firstly, the minimal path approach alone can only find a minimizer of an edge functional, with one or several(s) fixed input end-point(s). Conversely, our model is suitable to any energy functional, which we prove by endowing it with edge-based or different regionbased energies, including the minimal variance of the Chan and Vese model [12]. We believe that describing the curve with geodesics is pertinent whatever the energy functional is. Indeed, whether the functional holds edge, region and/or even shape prior terms, the major part of the final curve will be located on more or less salient edges. In comparison to snakes driven by gradient descent, the use of search windows significantly reduces sensitivity to erroneous local minima and energy weights tuning.

\section{Background}

\subsection{Parametric and geodesic active contours}

The active contour model is represented as a plane curve $\Gamma$ with $C^{2}$ position vector $\mathbf{c}(u)=[x(u) y(u)]$. Segmentation of an object of interest is performed by finding the curve minimizing an energy functional $E$, which has the general form:

$$
E(\Gamma)=\int_{0}^{1} \mathcal{L}\left(\mathbf{c}, \mathbf{c}^{\prime}, \mathbf{c}^{\prime \prime}\right) d u
$$

where $\mathcal{L}$ is usually made up of internal terms regularizing the curve and external terms attaching the curve to image data. According to calculus of variations, the following variational derivative vanishes if the curve is a local minimizer of $E$ :

$$
\frac{\delta E}{\delta \Gamma}=\frac{\partial \mathcal{L}}{\partial \mathbf{c}}-\frac{d}{d u}\left\{\frac{\partial \mathcal{L}}{\partial \mathbf{c}^{\prime}}\right\}+\frac{d^{2}}{d u^{2}}\left\{\frac{\partial \mathcal{L}}{\partial \mathbf{c}^{\prime \prime}}\right\}
$$


Curve evolution is usually performed by gradient descent, taking the opposite variational derivative as a descent direction. Given an image $I$ defined over $\mathcal{D} \in \mathbb{R}^{2}$, they use the following edge indicator $g$, which is a decreasing function of gradient magnitude:

$$
g(\mathbf{x})=\frac{1}{1+\left\|\nabla G_{\sigma} * I(\mathbf{x})\right\|}
$$

where the image is convolved with the derivative of a gaussian of standard deviation $\sigma$. The original parametric snake [1] has the following energy and variational derivative:

$$
\begin{aligned}
\mathcal{L}_{\text {snake }}\left(\mathbf{c}, \mathbf{c}^{\prime}, \mathbf{c}^{\prime \prime}\right) & =\frac{1}{2}\left(\alpha\left\|\mathbf{c}^{\prime}\right\|^{2}+\beta\left\|\mathbf{c}^{\prime \prime}\right\|^{2}\right)+g(\mathbf{c}) \\
\frac{\delta E_{\text {snake }}}{\delta \Gamma} & =-\alpha \mathbf{c}^{\prime \prime}+\beta \mathbf{c}^{\prime \prime \prime \prime}+\nabla g
\end{aligned}
$$

The energy functional of the snake is dependent on parameterization. This has an impact on discretization, since the energy varies in terms of sampling when the contour is implemented as a polygonal curve. The geodesic active contour (GAC) [4][13] solves the parameterization issue by introducing an intrinsic energy functional, weighting the edge indicator by length element $\left\|\mathbf{c}^{\prime}\right\|$ :

$$
\begin{aligned}
\mathcal{L}_{\mathrm{GAC}}\left(\mathbf{c}, \mathbf{c}^{\prime}, \mathbf{c}^{\prime \prime}\right) & =g(\mathbf{c})\left\|\mathbf{c}^{\prime}\right\| \\
\frac{\delta E_{\mathrm{GAC}}}{\delta \Gamma} & =(\langle\nabla g, \mathbf{n}\rangle-\kappa g) \mathbf{n}
\end{aligned}
$$

where $\mathbf{n}$ and $\kappa$ are the unit inward normal vector and curvature, respectively. Hence, the flow resulting from the geometric energy also holds a regularization term. This model lends itself to level set implementation, allowing topology changes.

Boundary-based active contours driven by gradient descent, whether parametric or geodesic, are relatively blind to neighboring structures. They are prone to getting trapped in erroneous local minima induced by noise and lack of contrast between objects. To increase the capture range of parametric contours, the balloon model introduced in [5] adds a bias force in the direction normal to the curve, so that this one can be inflated or deflated towards object boundaries. In level set implementations, the bias is commonly referred to as the advection term [3]. However, despite such techniques, gradient descent may still cause the contour to miss or pass through significant boundaries. The minimal path method addresses this issue by finding a global minimum of the energy.

\subsection{Minimal paths}

The minimal path approach by Cohen and Kimmel [10] aims at finding curves of minimal lengths in a Riemannian space endowed with an heterogeneous isotropic metric. The length of path $\mathcal{C}$ is:

$$
L(\mathcal{C})=\int_{0}^{1} \tilde{P}(\mathcal{C}(s)) d s=\int_{0}^{1} \tilde{P}(\mathcal{C}(u))\left\|\mathcal{C}^{\prime}(u)\right\| d u
$$

where $s$ is the arc length. Potential $\tilde{P}$, which defines the isotropic metric, should be chosen according to the application. Curves located on image boundaries are 
detected by using an edge-dependent potential $\tilde{P}(\mathbf{x})=w+g(\mathbf{x})$, where $w$ is a regularizing constant. Hence the cost of $\mathcal{C}$ may be rewritten using euclidean length:

$$
L(\mathcal{C})=\int_{0}^{1}(w+g(\mathcal{C}(s))) d s=w L_{\text {euclidean }}(\mathcal{C})+\int_{0}^{1} g(\mathcal{C}(s)) d s
$$

With respect to the energy functional to be minimized, the minimal path approach is similar to the geodesic active contour model, as can be seen in term $\mathcal{L}_{\mathrm{GAC}}$ of eq. 4 . However, the minimal path has the avantageous difference of reaching the global minimum of the energy, given two fixed end-points $\mathbf{x}_{0}$ and $\mathbf{x}_{1}$. Starting from point $\mathbf{x}_{0}$, the minimal action map $\mathcal{U}_{0}$ should be calculated. It corresponds to the minimal cost integrated along a path starting at $\mathbf{x}_{0}$ and ending at $\mathbf{x}$ :

$$
\mathcal{U}_{0}(\mathbf{x})=\inf _{\mathcal{C}}\{L(\mathcal{C})\} \text { s.t. }\left\{\begin{array}{l}
\mathcal{C}(0)=\mathbf{x}_{0} \\
\mathcal{C}(1)=\mathbf{x}
\end{array}\right.
$$

The action map $\mathcal{U}_{0}$ is the viscosity solution of the Eikonal equation:

$$
\|\nabla U(\mathbf{x})\|=\tilde{P}(\mathbf{x})
$$

with initial condition $U\left(\mathbf{x}_{0}\right)=0$. This allows $\mathcal{U}_{0}$ to be computed by the Fast Marching method [11], which is similar in principle to Dijkstra's graph search algorithm. Unlike the latter, it does not suffer from metrication errors and is thus consistent with the continuous problem. Using a binary heap structure to store candidate pixels, the Fast Marching algorithm computes the minimal action map in $O(N \log N)$ operations, where $N$ is the number of points. Once the action map has been computed, the geodesic $\gamma$, i.e. the path of minimal action linking a point $\mathbf{x}_{1}$ to $\mathbf{x}_{0}$, is found by back-propagation starting from $\mathbf{x}_{1}$. Gradient descent of the action map is performed until $\mathbf{x}_{0}$ is reached:

$$
\frac{\partial \gamma(u)}{\partial u}=-\nabla \mathcal{U}_{0}(\gamma(u))
$$

In its initial formulation, the minimal path method determines an open curve between two fixed end-points. It is also able to find closed contours by providing only one point on the final contour and detecting a saddle point on the minimal action map [10].

\subsection{Greedy algorithm}

Along with dynamic programming [6][7], greedy methods deal with discrete energy functionals. The greedy algorithm for active contours, as developed in [8], seeks for a minimizer of the energy by means of a set of local optimizations. It is only applicable on explicit implementations, where the contour is represented as a polygon with $n$ vertices $\left\{\mathbf{v}_{i}\right\}_{1 \leq i \leq n}$. The total energy is considered as a sum of vertex energies:

$$
E(\Gamma)=\sum_{i=1}^{n} E_{\mathrm{vertex}}\left(\mathbf{v}_{i}\right)
$$

where $E_{\text {vertex }}$ is the discretization of the energy at a given vertex, using finite differences. Considering the snake term $\mathcal{L}_{\text {snake }}$ of eq. 3 , it comes:

$$
E_{\text {vertex }}\left(\mathbf{v}_{i}\right)=\frac{1}{2}\left(\alpha\left\|\mathbf{v}_{i}-\mathbf{v}_{i-1}\right\|^{2}+\beta\left\|\mathbf{v}_{i+1}-2 \mathbf{v}_{i}-\mathbf{v}_{i-1}\right\|^{2}\right)+g\left(\mathbf{v}_{i}\right)
$$


Vertices are successively moved in order to minimize their own energies. At each iteration, a square window of width $m$ is considered around the current vertex. The energy of the latter is computed at each tested position $\tilde{\mathbf{v}}_{i}$ in the window. The vertex is then moved to the position leading to the lowest energy, which is summarized by the evolution scheme, at iteration $t$ :

$$
\mathbf{v}_{i}^{(t+1)}=\underset{\tilde{\mathbf{v}}_{i} \in \mathcal{W}\left(\mathbf{v}_{i}^{(t)}\right)}{\operatorname{argmin}} E_{\text {vertex }}\left(\tilde{\mathbf{v}}_{i}\right)
$$

where $\mathcal{W}(\mathbf{x})$ is the window centered at point $\mathbf{x}$. The initial greedy algorithm [8] performs in $O\left(\mathrm{~nm}^{2}\right)$ operations. The window size has an obvious impact on computational cost, but also on convergence abilities. Indeed, the contour can capture farther structures as the window is larger. Recently, an evolution strategy based on an alternation of several windows was proposed in [9] to speed up evolution. The greedy algorithm is by essence a discrete optimization heuristic. The formulation of the variational derivative is not used and continuous calculus of variations is thus not necessary.

\section{The geodesically linked active contour}

We develop an approach taking advantage of above described methods. Our geodesically linked active contour is based simultaneously on an explicit implementation of active contours, minimal paths and the greedy algorithm. Basically, we deal with an evolving explicit closed curve, allowing initialization inside or around the target object without providing fixed points. Minimal paths coupled with a geometric energy functional allows a parameterization-free handling of the contour. The use of the greedy algorithm, as opposed to gradient descent, guarentees better robustness to local minima.

\subsection{Minimal paths to connect vertices}

Let us consider a set of $n$ linked vertices $S=\left\{\mathbf{v}_{i}\right\}_{1 \leq i \leq n}$. We denote as $\gamma_{i}(u)=$ $\left[x_{i}(u) y_{i}(u)\right]$ the geodesic path connecting $\mathbf{v}_{i}$ to $\mathbf{v}_{i+1}$ :

$$
\gamma_{i}=\underset{\mathcal{C}}{\operatorname{argmin}}\{L(\mathcal{C})\} \text { s.t. }\left\{\begin{array}{l}
\mathcal{C}(0)=\mathbf{v}_{i} \\
\mathcal{C}(1)=\mathbf{v}_{i+1}
\end{array}\right.
$$

where the cost functional $L$ is defined in eq. 5. At every step of the evolution algorithm, the set of geodesics $\left\{\gamma_{i}\right\}_{1 \leq i \leq n}$ describes a closed piecewise differentiable contour $\Gamma$, which euclidean length is:

$$
L_{\text {euclidean }}(\Gamma)=\sum_{i=1}^{n} \int_{0}^{1}\left\|\gamma_{i}^{\prime}(u)\right\| d u
$$

One may note that a concatenation of geodesics $\gamma_{i}$ is not a geodesic itself, since it is forced to pass through given points. To some extent, curve $\Gamma$ may be considered as a piecewise minimizer of an edge-based functional. If a uniform potential $\tilde{P}(\mathbf{x})=1$ was chosen, the geodesics would become straight lines of equation

$$
\gamma_{i}(u)=(1-u) \mathbf{v}_{i}+u \mathbf{v}_{i+1}, u \in[0,1]
$$

in which case $\Gamma$ would represent a polygon. Fig. 1 depicts geodesically linked contours with uniform potential and edge-based potential (dark smooth lines 
represent high image gradient areas). As described in section 2.2, path $\gamma_{i}$ is determined by gradient descent of the minimal action map $\mathcal{U}_{i+1}$ of origin $\mathbf{v}_{i+1}$. Given start point $\mathbf{v}_{i+1}$, the Fast Marching algorithm [11] allows to specify one or several end points (in our case $\mathbf{v}_{i}$ ) so that propagation can be stopped when $\mathbf{v}_{i}$ is reached. This prevents the whole image from being visited by the Fast Marching and saves computational time.

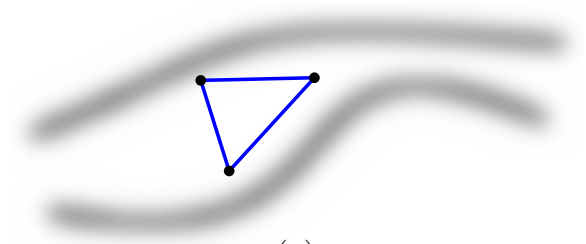

(a)

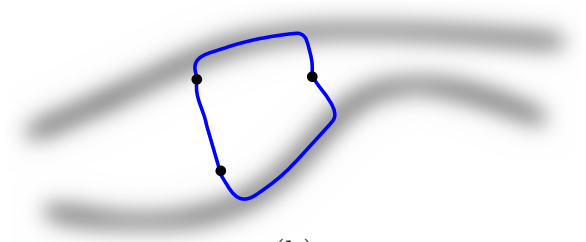

(b)

Fig. 1. Vertices linked by geodesics with uniform potential (a) and edge-based potential (b)

In the case of edge-based segmentation, the interest of describing the evolving contour with geodesics is obvious. Indeed, in the end of deformation, the geodesics fit the actual boundaries of the sought object. On the other hand, in the case of region-based segmentation, image edges are not explicitly searched. However, we believe that linking vertices with geodesics is relevant for any usual segmentation criterion. We may assume that the final contour should be partially located on more or less salient boundaries, whatever energy functional is optimized. In subsequent sections, we formulate three energies independently implemented on the geodesically linked active contour, namely the edge, region and narrow band region energies. Before, we recall Green's theorem, which we use to convert domain integrals into boundary integrals. For every region $R$ and real-valued function $f$ over $\mathbb{R}^{2}$, we have:

$$
\int_{R} f(\mathbf{x}) d \mathbf{x}=\int_{\partial R} P d x+Q d y
$$

where $[P(\mathbf{x}) Q(\mathbf{x})]$ is a continuously differentiable vector field such that:

$$
Q(x, y)=\frac{1}{2} \int_{-\infty}^{x} f(t, y) d t \quad P(x, y)=-\frac{1}{2} \int_{-\infty}^{y} f(x, t) d t
$$

The theorem expects that $\partial R$ should be at least piecewise smooth, it is thus applicable to the geodesically linked active contour. For instance, to express the area of region $R_{\text {in }}$ enclosed by $\Gamma$, we consider eq. 9 with $f(\mathbf{x})=1$ :

$$
\left|R_{\text {in }}\right|=\frac{1}{2} \sum_{i=1}^{n} \int_{0}^{1} x_{i}(u) y_{i}^{\prime}(u)-x_{i}^{\prime}(u) y_{i}(u) d u
$$

\subsection{Edge energy}

Boundary-based segmentation is performed by minimizing an edge energy. The edge indicator function $g$ is integrated along geodesics. In order not to penalize 
lengthy contours, the edge energy is normalized by euclidean length:

$$
E_{\text {edge }}(\Gamma)=\frac{1}{L_{\text {euclidean }}(\Gamma)} \sum_{i=1}^{n} \int_{0}^{1} g\left(\gamma_{i}(u)\right)\left\|\gamma_{i}{ }^{\prime}(u)\right\| d u
$$

Note that according to eq. 6 , the integral of $g$ along $\gamma_{i}$ equals $\mathcal{U}_{i+1}\left(\mathbf{v}_{i}\right)$ minus the euclidean length $L_{\text {euclidean }}\left(\gamma_{i}\right)$. Hence, once the action maps have been computed, the edge indicator does not need to be summed over geodesics again. With the edge energy alone, if the search space of vertex coordinates is too small, the contour fails at capturing actual boundaries when initialized far from them. To increase the capture range, we add an area-dependent term, which minimization acts like a balloon force [5]:

$$
E_{\text {balloon }}(\Gamma)=\frac{|\mathcal{D}|-\left|R_{\text {in }}\right|}{|\mathcal{D}|}
$$

where $|\mathcal{D}|$ is the image area. In that case, the total energy is a weighted sum of edge and balloon energies.

\subsection{Region energy}

The increasing use of region terms has proven to overcome limitations of edgebased only models, especially when dealing with data sets suffering from noise and lack of contrast between neighboring structures. Classical region-based deformable models segment images according to statistical data computed over the object of interest and the background. Image partitions should be uniform in terms of pixel intensities or higher level features like texture descriptors. We rely on the intensity variance, which is close to the two-phase Mumford-Shah segmentation model by Chan and Vese [12]. The average intensity in the inner region is expressed using Green's theorem:

$$
\mu\left(R_{i n}\right)=\frac{1}{\left|R_{i n}\right|} \int_{R_{i n}} I(\mathbf{x}) d \mathbf{x}=\frac{1}{\left|R_{i n}\right|} \sum_{i=1}^{n} \int_{0}^{1} x_{i}^{\prime} P\left(\gamma_{i}\right)+y_{i}^{\prime} Q\left(\gamma_{i}\right) d u
$$

where $P$ and $Q$ are the summed intensities (see template formulas in eq. 10). Then, the inner intensity variance is:

$$
\sigma^{2}\left(R_{i n}\right)=\frac{1}{\left|R_{i n}\right|} \int_{R_{i n}}\left(I(\mathbf{x})-\mu\left(R_{i n}\right)\right)^{2} d \mathbf{x}=\frac{1}{\left|R_{i n}\right|} \int_{R_{i n}} I^{2}(\mathbf{x}) d \mathbf{x}-\mu\left(R_{i n}\right)^{2}
$$

where the integral of squared intensities may also be expanded according to Green's theorem. Corresponding quantities on the outer region may be expressed using relation

$$
\int_{R_{\text {out }}} f(\mathbf{x}) d \mathbf{x}=\int_{\mathcal{D}} f(\mathbf{x}) d \mathbf{x}-\int_{R_{\text {in }}} f(\mathbf{x}) d \mathbf{x}
$$

\subsection{Narrow band region energy}

The ideal case of uniform regions is rarely encountered in real applications, as the background usually contains structures of various intensities. Hence, strict homogeneity is not necessarily a desirable property. In order to account for spatially varying intensity, local statistics in region-based segmentation have 
emerged recently [14] [15]. The narrow band principle, which has proven its efficiency in the evolution of level sets [3], is used in our approach to formulate a local region term $[16]$.

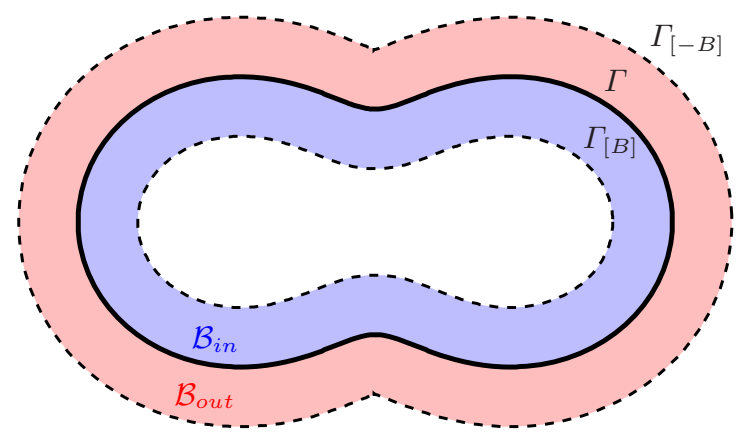

Fig. 2. Inner and outer bands for narrow band region energy

Instead of dealing with whole domains $R_{\text {in }}$ and $R_{\text {out }}$, we consider an inner band $\mathcal{B}_{\text {in }}$ and an outer band $\mathcal{B}_{\text {out }}$ in the vicinity of the contour, as depicted in fig. 2. The narrow band region energy is the intensity variance over the bands:

$$
E_{\text {band }}(\Gamma)=\sigma^{2}\left(\mathcal{B}_{\text {in }}\right)+\sigma^{2}\left(\mathcal{B}_{\text {out }}\right)
$$

Our narrow band region energy is based on parallel curves [17]. We define curve $\gamma_{[B]_{i}}$ as a parallel curve of $\gamma_{i}$ :

$$
\gamma_{[B]_{i}}(u)=\gamma_{i}(u)+B \mathbf{n}_{i}(u)
$$

where $B$ is the user-defined band thickness, constant along the curve, and $\mathbf{n}_{i}$ is the inward unit normal to geodesic $\gamma_{i}$. Hereafter, we will use the index $[B]$ to denote all quantities related to the parallel curve. Bands $\mathcal{B}_{\text {in }}$ and $\mathcal{B}_{\text {out }}$ are bounded by parallel curves of the $n$ geodesics $\gamma_{i}$, respectively $\gamma_{[B]_{i}}$ and $\gamma_{[-B]_{i}}$. We assume that geodesics are smooth enough so that their parallel curves do not self-intersect nor exhibit singularities. An important property resulting from the definition in eq. 11 is that the velocity vector of the parallel curve can be expressed as a function of the velocity vector of the initial curve, as well as its curvature and normal. Using the identity $\mathbf{n}_{i}{ }^{\prime}=-\kappa_{i} \gamma_{i}{ }^{\prime}$, we have:

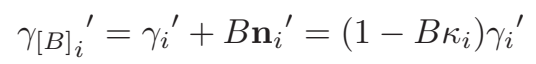

By a change of variable, an integral over inner band $\mathcal{B}_{\text {in }}$ may be expressed explicitly in terms of the curve and band thickness:

$$
\int_{\mathcal{B}_{i n}} f(\mathbf{x}) d \mathbf{x}=\sum_{i=1}^{n} \int_{0}^{1} \int_{0}^{B} f\left(\gamma_{i}+b \mathbf{n}_{i}\right)\left\|\gamma_{i}^{\prime}\right\|\left(1-b \kappa_{i}\right) d b d u
$$

We use the template formula in eq. 13 to express the mean and variance of intensities in the inner band:

$$
\begin{gathered}
\mu\left(\mathcal{B}_{i n}\right)=\frac{1}{\left|\mathcal{B}_{i n}\right|} \sum_{i=1}^{n} \int_{0}^{1} \int_{0}^{B} I\left(\gamma_{i}+b \mathbf{n}_{i}\right)\left\|\gamma_{i}^{\prime}\right\|\left(1-b \kappa_{i}\right) d b d u \\
\sigma^{2}\left(\mathcal{B}_{i n}\right)=\frac{1}{\left|\mathcal{B}_{i n}\right|} \sum_{i=1}^{n} \int_{0}^{1} \int_{0}^{B}\left(I\left(\gamma_{i}+b \mathbf{n}_{i}\right)-\mu\left(\mathcal{B}_{i n}\right)\right)^{2}\left\|\gamma_{i}^{\prime}\right\|\left(1-b \kappa_{i}\right) d b d u
\end{gathered}
$$


and similarly for the outer band, replacing $b$ with $-b$.

\subsection{Evolution with greedy algorithm}

Vertices should be moved in order to minimize the selected energy. This is usually performed with gradient descent of the Euler-Lagrange equation. In our case, it is difficult to differentiate $E_{\text {edge }}, E_{\text {region }}$ or $E_{\text {band }}$ with respect to a given vertex $\mathbf{v}_{i}$, since these energies depend on geodesics to $\mathbf{v}_{i}$ (see eq. (8)). The greedy algorithm presented in section 2.3 provides us a way to evolve vertices without differentiating the energy.

Motion of curve points can always be decomposed into normal and tangential components. While the geometry of the curve is modified by normal displacements, tangential motion only affects curve parameterization [4]. Since the distribution of vertices along the contour can be updated with a resampling technique, we only consider normal displacement in the greedy evolution. We define a normal-oriented window $\mathcal{W}_{\mathrm{N}}$ of length $m$ centered at vertex $\mathbf{v}_{i}$ :

$$
\mathcal{W}_{\mathrm{N}}\left(\mathbf{v}_{i}\right)=\left\{\mathbf{v}_{i}+k \mathbf{n}_{\mathbf{v}_{i}} \mid k \in \llbracket-\frac{m}{2}, \frac{m}{2} \rrbracket\right\}
$$

where $\mathbf{n}_{\mathbf{v}_{i}}$ is the inward unit normal vector, estimated by finite difference using the second and next-to-last points of geodesics $\gamma_{i}$ and $\gamma_{i+1}$, respectively. Since steps between successive points in the window are integers, the window may be computed using a Bresenham-like algorithm.

Greedy evolution is performed by moving vertex $\mathbf{v}_{i}$ to the position in the window which corresponding geodesically linked contour has the smallest energy. Let us consider a test position $\tilde{\mathbf{v}}_{i}$ belonging to the window. The associated geodesics $\tilde{\gamma}_{i-1}$ and $\tilde{\gamma}_{i}$ link it to the neighbors of $\mathbf{v}_{i}$, as depicted in fig. 3 . The energy of the corresponding geodesically linked contour

$$
\tilde{\Gamma}=\left\{\gamma_{1}, \ldots, \gamma_{i-2}, \tilde{\gamma}_{i-1}, \tilde{\gamma}_{i}, \gamma_{i+1}, \ldots, \gamma_{n}\right\}
$$

is computed and compared to the energy of the initial contour $\Gamma$. All window points are tested in this way, so that the evolution scheme at iteration $t$ is:

$$
\mathbf{v}_{i}^{(t+1)}=\underset{\tilde{\mathbf{v}}_{i} \in \mathcal{W}_{\mathrm{N}}\left(\mathbf{v}_{i}^{(t)}\right)}{\operatorname{argmin}} E(\tilde{\Gamma})
$$

where $E$ is one of the previously described energies. If we consider set $\mathcal{H}=$ $\{1, \ldots, i-2\} \cup\{i+1, \ldots n\}$ holding indices of geodesics not influenced by a modification on $\mathbf{v}_{i}$, all quantities involved in the energies are written with constant and variable parts with respect to $\tilde{\mathbf{v}}_{i}$. For instance, the area of the tested inner region is decomposed:

$$
\begin{aligned}
\left|\tilde{R}_{i n}\right|= & \sum_{j \in \mathcal{H}} \int_{0}^{1} x_{j}(u) y_{j}^{\prime}(u)-x_{j}^{\prime}(u) y_{j}(u) d u \\
& +\int_{0}^{1} \tilde{x}_{i-1} \tilde{y}_{i-1}^{\prime}-\tilde{x}_{i-1}^{\prime} \tilde{y}_{i-1} d u+\int_{0}^{1} \tilde{x}_{i} \tilde{y}_{i}^{\prime}-\tilde{x}_{i}^{\prime} \tilde{y}_{i} d u
\end{aligned}
$$

This implies that the part of energies invariant with respect to $\tilde{\mathbf{v}}_{i}$ need to be computed only once, before moving $\mathbf{v}_{i}$. Finally, once all vertices have been treated, 
resampling may be performed to maintain consistent distribution of vertices along the curve.

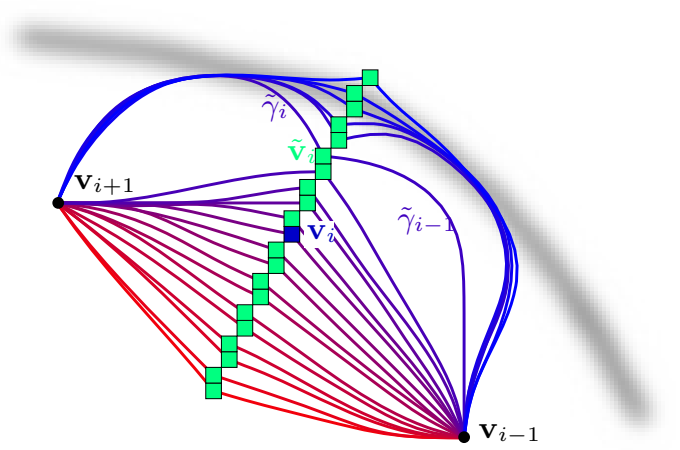

Fig. 3. Geodesics linking neighboring vertices to points in search window

\section{Experiments}

We tested the geodesically linked active contour with the three different energy configurations (edge+balloon, region and narrow band region). A comparison with a parametric snake endowed with the same energies is provided. The snake was initialized as a small circle inside the area of interest, far from the target boundaries. Similarly, the initial vertices of our model are sampled on a circle. Results are shown in fig. 4. The geodesically linked active contour is drawn with blue edges whereas the parametric contour appears in red. For each row, columns (a) and (b) represents the initial and final states of the geodesically linked active contour, respectively. Columns (c) and (d) represent the same states for the snake.

For all experiments, the regularization weight $w$ was set to 0.25 , which achieved sufficient regularization for all tested images. The size of the window was $m=50$ and the maximal inter-vertex distance for resampling was set to 20 . The image in row 1, which was segmented using the edge energy, depicts the gapclosing ability of the model. The geodesically linked active contour managed to pass through false edges and reach actual boundaries. Thanks to the large search window, it turned out to be rather unsensitive to balloon strength, as values for coefficient $\alpha$ in the range $[0.1,4]$ were suitable. On the other hand, the balloon coefficient has a strong influence on the gradient descent-driven parametric snake, which yields difficult parameter tuning. Actually, it was not possible to find a correct balloon weight allowing to jump false edges while stabilizing on real ones. The image in row 2, which was segmented using the region energy, depicts a similar phenomenon. The geodesically linked contour does not get trapped in small gaps in the region, which could present an interest for segmentation of partially occluded objects.

Row 3 depicts a MRI of the heart left ventricle, which was used to put the narrow band region energy into application. The band thickness $B$ is an 
important parameter. Apart from its impact on the algorithmic complexity computing intensity means and variances on the bands takes at least $O(n B)$ operations - it controls the trade-off between local and global features around the object. If $B=1$, the region energy is as local as an edge term. The main image property having an effect on the minimal band thickness is the edges sharpness. Indeed, the deformable curve needs a larger band as the boundaries of the target object are fuzzy. However, $B=10$ was a suitable value in our experiments. Note that we depict the state of the parametric snake before self-collision. One may note that an unconstrained region-based level set method would also properly segment images in row 2 and 3 . However, this remark should be moderated by the fact our model is dedicated to applications where topology preservation is needed.

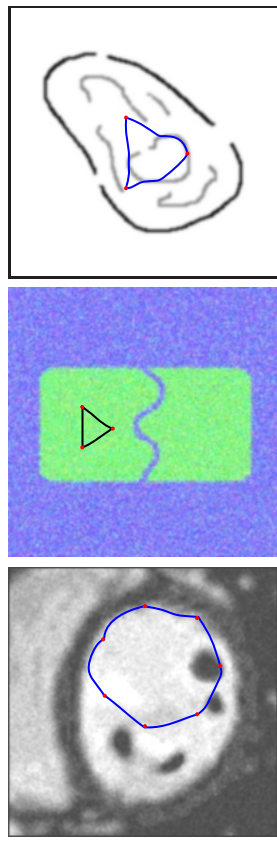

(a)
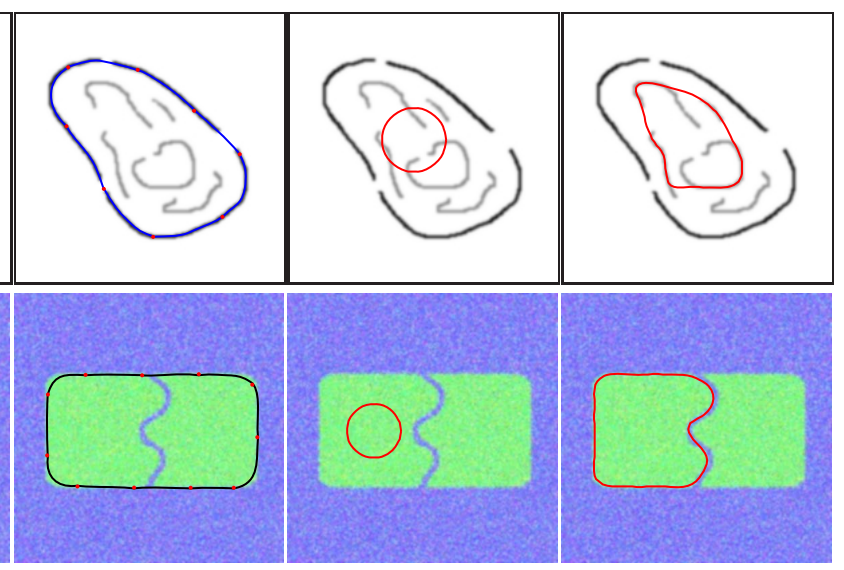

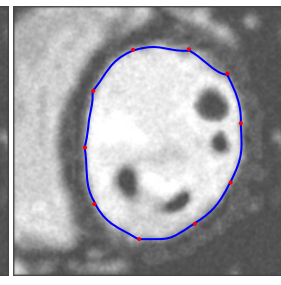

(b)

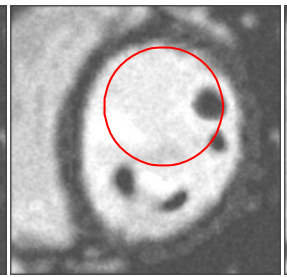

(c)

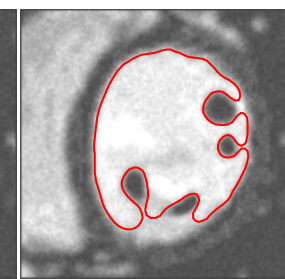

(d)

Fig. 4. Segmentation of left ventricle: initialization (a) and final location (b) of the geodesically linked active contour, initialization (c) and final location (d) of the parametric contour

\section{Conclusion and perspectives}

We proposed the geodesically linked active contour model for image segmentation. The model lies on an explicitly implemented curve moved by an evolution method based on minimal paths and a greedy algorithm. Linking curve points with geodesics solves parameterization issues and allows the contour to fit the most salient boundaries at every step of deformation. Displacing vertices according to a greedy search ensured lower sensitivity to erroneous local minima than 
usual gradient descent of the energy. The model was endowed with edge and region energies and was validated on a few datasets. Further work may focus on developing an adaptive search window for greedy evolution. Currently, the window length is constant whatever the values of energies or the previous positions of vertices. We believe the algorithm could be improved by adapting the window length with respect to these properties, in order to avoid visiting positions that would not seemingly minimize the energy.

\section{References}

1. Kass, M., Witkin, A., Terzopoulos, D.: Snakes: active contour models. International Journal of Computer Vision 1(4) (1988) 321-331

2. Osher, S., Sethian, J.: Fronts propagation with curvature-dependent speed: algorithms based on Hamilton-Jacobi formulations. Journal of Computational Physics 79 (1988) 12-49

3. Malladi, R., Sethian, J., Vemuri, B.: Shape modeling with front propagation: a level set approach. IEEE Transactions on Pattern Analysis and Machine Intelligence 17(2) (1995) 158-175

4. Caselles, V., Kimmel, R., Sapiro, G.: Geodesic active contours. International Journal of Computer Vision 22(1) (1997) 61-79

5. Cohen, L.: On active contour models and balloons. Computer Vision, Graphics, and Image Processing: Image Understanding 53(2) (1991) 211-218

6. Amini, A., Weymouth, T., Rain, R.: Using dynamic programming for solving variational problems in vision. IEEE Transactions on Pattern Analysis and Machine Intelligence 12(9) (1990) 855-867

7. Geiger, D., Gupta, A., Luiz, A., Vlontzos, J.: Dynamic programming for detecting, tracking, and matching deformable contours. IEEE Transactions on Pattern Analysis and Machine Intelligence 17(3) (1995) 294-302

8. Williams, D., Shah, M.: A fast algorithm for active contours and curvature estimation. Computer Vision, Graphics, and Image Processing: Image Understanding 55(1) (1992) 14-26

9. Sakalli, M., Lam, K.M., Yan, H.: A faster converging snake algorithm to locate object boundaries. IEEE Transactions on Image Processing 15(5) (2006) 11821191

10. Cohen, L., Kimmel, R.: Global minimum for active contour models: a minimal path approach. International Journal of Computer Vision 24(1) (1997) 57-78

11. Sethian, J.: A fast marching level set method for monotonically advancing fronts. Proceedings of the National Academy of Science 93(4) (1996) 1591-1595

12. Chan, T., Vese, L.: Active contours without edges. IEEE Transactions on Image Processing 10(2) (2001) 266-277

13. Kichenassamy, S., Kumar, A., Olver, P.and Tannenbaum, A., Yezzi, A.: Gradient flows and geometric active contour models. In: IEEE International Conference on Computer Vision (ICCV), Boston, MA, USA (1995) 810-815

14. Piovano, J., Rousson, M., Papadopoulo, T.: Efficient segmentation of piecewise smooth images. In: International Conference on Scale Space and Variational Methods in Computer Vision (SSVM), Ischia, Italy (2007) 709-720

15. Lankton, S., Tannenbaum, A.: Localizing region-based active contours. IEEE Transactions on Image Processing 17(11) (2008) 2029-2039

16. Mille, J., Boné, R., Cohen, L.: Region-based 2D deformable generalized cylinder for narrow structures segmentation. In: European Conference on Computer Vision (ECCV). Volume 5303 of LNCS., Marseille, France, Springer (2008) 392-404

17. Farouki, R., Neff, C.: Analytic properties of plane offset curves. Computer Aided Geometric Design 7(1-4) (1990) 83-99 